\title{
USE OF A WEB-BASED SUPPORT DURING A RANDOMISED CLINICAL TRIAL ON FIRST-EPISODE PSYCHOSIS: PRELIMINARY ACTIVITY DATA
}

Á. Orosa-Duarte ${ }^{1}$, Á. de Diego ${ }^{1}$, A. Muñoz $\mathrm{S}^{1,2}$, R. Mediavilla²,3, M.P. Vidal-Villegas ${ }^{1}$, N. Fernández ${ }^{1}$, Á. García ${ }^{1}$, G. Kollias ${ }^{1}$, J. M. Pastor ${ }^{1}$, G. Lahera ${ }^{4,5}$, E. Román ${ }^{1}$, P. Sánchez ${ }^{1}$, I.I. Louzao ${ }^{1}$, Á. Palao ${ }^{1,2}$, B. Rodríguez-Vega ${ }^{1,2}$, C. Bayón ${ }^{1,2}$, L. Gotor ${ }^{1}$, E. Jiménez ${ }^{1}$, I. Millán ${ }^{1}$, M.F. Bravo ${ }^{1,2}$.

1 La Paz University Hospital, Psychiatry-Clinical Psychology and Mental Health, Madrid, Spain

2 Hospital La Paz Institute for Health Research (IdiPAZ), Psychiatry and Mental Health Group, Madrid, Spain.

3 National University of Distance Education UNED, Psychology, Madrid, Spain.

4 University of Alcala, Faculty of Medicine. Psychiatry Department, Alcala de Henares, Spain.

5 CIBERSAM, Mental Health, Madrid, Spain.

\section{Background}

Difficulties in social functioning are common among people with schizophrenia-spectrum disorders (SSD) (Palumbo et al., 2015). Social cognition is a core predictor of social functioning (Green et al., 2015), but no mindfulness-based interventions (MBIs) targeting aspects of social cognition have been developed for people with psychosis.

SocialMind is a Mindfulness-based program oriented to improve social cognition. It has shown to be safe for people with schizophreniaspectrum disorders in a feasibility trial.

\section{Objective}

To analyse participants' activity on a website created as support for an ongoing clinical trial among people with first-episode psychosis.

\section{Material and methods}

We designed a randomised controlled trial to compare this program versus a psychoeducation intervention. A website was created, presenting either complementary Mindfulness material (homework and guided meditation clips) or psychoeducation content. Participants assisted group sessions and got a personal ID to access the corresponding website content. We collected activity data through Google Analytics obtaining descriptive measures.

\begin{tabular}{|c|c|c|c|}
\hline User ID & Sessions & Average session \\
duration & Device \\
\hline pc-23 & 10 & $6^{\prime} 10^{\prime \prime}$ & Desktop + Mobile \\
\hline pc-12 & 4 & $1^{\prime} 50^{\prime \prime}$ & Mobile \\
\hline pc-21 & 3 & $11^{\prime} 45^{\prime \prime}$ & Tablet \\
\hline pc-17 & 1 & $2^{\prime} 10^{\prime \prime}$ & Mobile \\
\hline pc-3 & 1 & $1^{\prime} 21^{\prime \prime}$ & Mobile \\
\hline pc-11 & 1 & $5^{\prime} 49^{\prime \prime}$ & Desktop \\
\hline pc-19 & 1 & $2^{\prime} 48^{\prime \prime}$ & Tablet \\
\hline
\end{tabular}

Table 1.

\section{Results and Conclusions}

An overview of activity data is shown in Figure 1. From October 2018 to February 2019, 7 out of 15 participants (47\%) had registered. 4 participants have accessed just once, while 3 participants have accessed 3-10 times. Detailed information may be found in Table 1.

Participants with first-episode psychosis can use a website as support to social cognition or psychoeducation training. In particular, the users from our sample preferred access through mobile devices. This kind of platform provides data that constitute new variables of interest. However, low usage rates of our website suggest the need to get users' feedback.

$$
47 \%
$$

users have registered

$$
\text { 1.200 }
$$

Mean session duration
Tablet

$29 \%$
Mobile Phone $43 \%$
Desktop $14 \% \quad 14 \%$

Figure 1. 